\title{
INVESTIGANDO O FENÔMENO DE COMPRAS COLETIVAS ON-LINE: FATORES QUE INFLUENCIAM A INTENSIDADE DAS COMPRAS
}

\author{
INVESTIGATING THE COLLECTIVE ONLINE \\ PURCHASE PHENOMENON: FACTORS AFFECTING \\ THE SHOPPING INTENSITY
}

\author{
Recebido 30-10-2012 \\ Aceito 11-06-2013 \\ Everton Roberto Comin ${ }^{1}$ \\ Rafael Teixeira ${ }^{2}$ \\ Juliana Durayski ${ }^{3}$ \\ Natália Gomes da Silva ${ }^{4}$ \\ Julio Vieira ${ }^{5}$
}

\section{RESUMO}

A compra coletiva on-line surgiu no Brasil há cerca de dois anos. Tem como objetivo vender produtos e serviços em massa com grandes descontos, exigindo um número mínimo de consumidores para atingir o volume pré-estipulado. Nesse contexto, este estudo visou investigar a relação entre a intensidade de compra coletiva on-line e o interesse do consumidor, a compra por impulso e a insegurança em relação ao site. A coleta dos dados, que englobou 143 respondentes, ocorreu por meio de um questionário contendo questões fechadas com múltiplas escolhas e escalas de concordância do tipo Likert de 5 pontos. As análises dos dados foram realizadas por meio de técnicas exploratórias preliminares, fatorial exploratória, confiabilidade e regressão logística. Os resultados sugerem que pessoas com maior interesse em compras coletivas têm maiores chances de realizar compras coletivas. Identificou-se que, quanto maior a insegurança em relação ao site, menor a chance de a pessoa realizar compras coletivas. Observou-se que não há uma relação positiva entre compra por impulso e compra coletiva. Entre as limitações da pesquisa, destaca-se o número de construtos e as características da amostra.

Palavras-chave: Intensidade; Compra coletiva on-line, Interesse, Impulso, Insegurança.

\footnotetext{
1 Possui graduação em Administração pelo Centro Universitário Univates, especialização em Gerenciamento de Projetos pela Universidade do Vale do Rio dos Sinos - UNISINOS e mestrado em Administração pela Universidade do Vale do Rio dos Sinos - UNISINOS. Atualmente é coordenador de Auditoria em Saúde na UNIMED. Lajeado, Rio Grande do Sul, Brasil. E-mail: everton.comin@gmail.com.

${ }^{2}$ Possui graduação em Administração pela Universidade Federal do Rio Grande do Sul - UFRGS, especialização em Marketing pela Fundação Getúlio Vargas - FGV, mestrado em Administração pela Universidade do Vale do Rio dos Sinos - UNISINOS e doutorado em Gestão pela Clemson University - Estados Unidos. Atualmente é professor em Administração na Universidade do Vale do Rio dos Sinos - UNISINOS. São Leopoldo, Rio Grande do Sul, Brasil. E-mail: teixeira.rafa@gmail.com.

${ }^{3}$ Possui graduação em Comunicação Social - Publicidade e Propaganda pela Escola Superior de Propaganda e Marketing ESPM, especialização em Econegócios e Gestão Socioambiental pela Universidade Federal do Rio Grande do Sul - UFRGS e mestrado em Administração pela Universidade do Vale do Rio dos Sinos - UNISINOS. Atualmente é pesquisadora na Universidade do Vale do Rio dos Sinos - UNISINOS. Porto Alegre, Rio Grande do Sul, Brasil. E-mail: durayskiju@hotmail.com.

${ }^{4}$ Possui graduação em Administração pela Universidade Federal do Rio Grande do Sul - UFRGS, especialização em Gestão Pública Eficaz pela Universidade Federal do Rio Grande do Sul - UFRGS e mestrado em Administração pela Universidade do Vale do Rio dos Sinos - UNISINOS. Atualmente é tutora na Universidade do Vale do Rio dos Sinos - UNISINOS. Porto Alegre, Rio Grande do Sul, Brasil. Email: ngsilva@terra.com.br.

${ }^{5}$ Possui graduação em Engenharia Mecânica pela Universidade Federal do Rio Grande do Sul - UFRGS, mestrado em Administração pela Universidade do Vale do Rio dos Sinos - UNISINOS
} 


\begin{abstract}
The collective online shopping is a recent phenomenon in Brazil whose objective is to sell products and services for a minimum number of consumers. This study aims to investigate whether the intensity of collective online shopping is influenced by the aspects consumer interest in online collective shopping, impulse buying and insecurity. The data collection was based on a questionnaire with closed questions with multiple choices and a 5-point Likert scale.The final sample is composed of 143 respondents. Data was analyzed through the following statistic techniques: exploratory data analysis, exploratory factor analysis, reliability analysis, and logistic regression. The results suggest that people more interest in collective shopping are more likely to make collective shopping. People who feel greater insecurity in navigating the website is less likely to make collective shopping. It was observed that there is no a positive relationship between impulse shopping and collective purchasing. Some limitations include the number of constructs and sample characteristics.
\end{abstract}

Keywords: Intensity, Collective online purchase, Interest, Impulse, Insecurity.

\title{
1 INTRODUÇÃO
}

Amplamente noticiado em 2010 na literatura de negócios, o fenômeno das compras coletivas on-line teve origem nos EUA, em 2008, com o surgimento do Groupon (STEINER, 2010) e, no Brasil, em 2010, com o lançamento do site Peixe Urbano (FAUST, 2010). Passados dois anos, em agosto de 2012, o mercado brasileiro contava com cerca de 800 empresas prestadoras desse tipo de serviço (COLDIBELI, 2012). Analistas de mercado apontam para um amadurecimento e uma consolidação do setor, que teve um faturamento de R\$ 731 milhões no primeiro trimestre de 2012 (COLDIBELI, 2012; FELIPINI, 2012). Devido a esses índices, os serviços de compras coletivas on-line têm chamado a atenção de empresas, empresários, pesquisadores, consumidores e outros interessados.

Por ser um tema contemporâneo, as pesquisas sobre compras coletivas on-line estão surgindo em periódicos acadêmicos somente nos últimos anos. Exemplos disso são os trabalhos de Dholakia (2010) e de Edelam et al. (2011), que abordam o uso do portal de compras Groupon pelos consumidores norte-americanos. No Brasil, foram identificados os estudos de Abreu e Sousa (2011), que fazem uma análise de caráter mais sociológico das compras coletivas, Gaudencio et. al. (2011) e Fronza e Freire (2012), que analisam o fenômeno a partir de uma perspectiva de estratégias de mercado. Outras pesquisas sobre o comportamento do consumidor on-line têm sido realizadas há certo tempo, inclusive no Brasil. Além disso, a literatura de negócios tem abordado o tema sob a perspectiva das empresas que estão oferecendo esse tipo de serviço, elucidando especialmente as estratégias de diferenciação em um mercado com tantos players atuando (FAUST, 2010; FEIJÓ, 2011; STEINER, 2010; PORTAL HSM, 2011). Percebe-se que o tema tem ganhado destaque no meio acadêmico e no meio executivo, demonstrando a relevância em aprofundar os conhecimentos sobre o assunto.

No sentido de proporcionar evidências empíricas que ajudem a entender o comportamento do consumidor brasileiro frente a esse fenômeno, este estudo tem caráter exploratório e busca contribuir para a compreensão da seguinte questão de pesquisa: qual a relação entre os construtos (i) interesse do consumidor por compras coletivas on-line, (ii) compra por impulso e (iii) insegurança em relação ao site e a variável intensidade de compra coletiva on-line? Dessa forma, objetiva-se verificar se existe alguma influência dessas variáveis na intensidade com que o consumidor faz uso desse tipo de serviço. Faz-se necessário explicitar que intensidade de compra é definida como o montante de compras coletivas on-line feitas por um comprador em um determinado período de tempo, medido, neste estudo, como uma variável binária que distingue os compradores de alta e baixa intensidade de compra. 
Entende-se que a decisão de compra no comércio eletrônico contempla diversas variáveis, as quais já foram apresentadas em distintos modelos teóricos (KOUFARIS, 2002; CHEUNG, CHAN E LIMAYEN, 2005; DENNIS ET AL., 2009; LIEBERMANN E STASHEVSKY, 2009); porém, para o caso desta pesquisa, optou-se por delimitar os construtos aos três anteriormente mencionados pelos seguintes motivos: i) o construto interesse por compras coletivas on-line procura mapear o quanto essa nova modalidade é de fato atrativa aos consumidores; ii) o construto impulso voltou a ser questionado na literatura de negócios, evocando a irracionalidade a que os consumidores podem estar sujeitos, tendo em vista os preços atraentes das ofertas e o curto prazo para adquiri -las; e iii) mesmo o comércio eletrônico estando em expansão, a segurança ainda mostra-se como umas das variáveis que mais inibe essa modalidade de comércio, conforme será exposto na seção 2 deste artigo. Em síntese, procurou-se, especialmente, averiguar se o que vem sendo vinculado na mídia e na literatura de negócios de fato pode ser diagnosticado em uma pesquisa científica.

Para o propósito deste estudo, compra coletiva on-line é definida como uma modalidade de comércio eletrônico que objetiva vender produtos e serviços em oferta para um número mínimo de consumidores. A compra coletiva on-line é nominada dessa forma porque as ofertas somente são finalizadas se o anunciante consegue concretizar a venda de uma quantidade mínima do que foi ofertado, ou seja, a venda só ocorre se houver várias pessoas que estejam dispostas a comprar as ofertas disponibilizadas.

Este trabalho representou uma tentativa de contribuição para o vasto e consolidado campo de conhecimentos sobre comportamento do consumidor, trazendo dados sobre construtos selecionados relativos à modalidade de compra coletiva on-line, a partir da proposição de um modelo de inter-relação entre esses construtos e a posterior coleta de dados empíricos. Pelo caráter eletivo dos construtos, assume-se a limitação dos resultados coletados.

\section{REFERENCIAL TEÓRICO}

Nesta seção são apresentados os aspectos teóricos revisados para elaboração deste estudo.

\subsection{Comportamento do Consumidor no Comércio On-line}

O comportamento do consumidor, ao fazer compras na internet, é, de certo modo, diferente do seu comportamento de compras fora da internet devido a fatores existentes em uma operação e inexistentes em outra. Conforme Liebermann e Stashevsky (2009), na compra tradicional, isto é, off-line, os consumidores são diretamente expostos aos itens, podendo utilizar-se especialmente dos sentidos (olfato, tato e visão) para realizar a compra. Já nas modalidades de comércio on-line, isso não ocorre, entretanto, "[...] a Internet pode fornecer informações mais detalhadas e atualizadas em tempo real (por exemplo, comparações de preços) para apoiar a tomada de decisão" (LIEBERMANN e STASHEVSKY, 2009, p. 317).

Lokken e colaboradores (2003), em pesquisa aplicada a professores e funcionários de uma universidade de cidade rural do sudeste dos Estados Unidos (130 participantes), identificaram como principais benefícios de compras on-line, em ordem crescente de importância: comentários e recomendações de especialistas; economia de tempo; conveniência; acesso a opiniões dos outros; ampla oferta de informações de fornecedores; nenhuma pressão de vendedores; e melhores preços. Como desvantagens percebidas pelos usuários que não realizam compras on-line (non-online shoppers), foram detectadas os seguintes aspectos: questões de segurança; satisfação com as compras locais; não ter acesso à internet em casa; ausência da demonstração 
de produto; ausência de informação sobre como comprar on-line; e questões de privacidade.

Cheung, Chan e Limayen (2005), a partir de uma revisão de 355 artigos publicados no período de 1994 a junho de 2005, propõem um modelo empírico integrado para o estudo do comportamento do consumidor on-line, estabelecendo que a compra deste consumidor está associada à intenção, adoção e continuidade - variáveis dependentes - dessa modalidade de comércio e que características individuais do consumidor (demográficas, estilo de vida, etc.), do produto/serviços (tipo do produto, preço, etc.), da mídia (interface, navegabilidade, confiança, segurança, etc.), do comerciante e dos intermediários (qualidade do serviço, reputação da marca, privacidade e controle de segurança, etc.) e as influências do ambiente (cultura, atenção, imagem, etc.) - variáveis independentes - são determinantes do comportamento do consumidor on-line.

Liebermann e Stashevsky (2009), em estudo sobre compras on-line com usuários de Israel em mercados em estágio inicial, em que a internet é relativamente nova, após revisão da literatura, propõem um modelo conceitual dos fatores que afetam as compras on-line nesse tipo de mercado. Tal modelo inclui aspectos como benefícios percebidos pelos clientes, custos de segurança, processo de compra, comportamento de uso da internet e exposição à informação. Esses autores aplicaram um questionário a 1080 empregados de aproximadamente 120 firmas israelenses, em fevereiro de 2004, e verificaram que o comprador on-line é alguém que percebe que os custos desse tipo de compra são relativamente baixos, reconhece os benefícios do comércio eletrônico, navega na internet muitas horas semanalmente, é relativamente um usuário veterano e usa a internet para uma variedade de atividades, incluindo o e-mail. Além disso, geralmente costuma ser do sexo masculino e mais velho, faz compras por telefone, não especialmente gosta de comprar, tende a ser o comprador da família e frequentemente usa a internet para pesquisar informações sobre produtos ou serviços.

\subsection{Compras Coletivas On-line}

A conceituação do fenômeno compras coletivas on-line, conforme observado nos artigos científicos estudados, ocorre, principalmente, como descrição do serviço a partir dos sites que os oferecem, como é o caso de Abreu e Sousa (2011, p. 1-2), que definem sites de compras coletivas como "aqueles que oferecem produtos ou serviços com exagerados descontos mediante, em geral, a adesão de um número mínimo de compradores", ou, então, de Fronza e Freire (2012), que destacam a exploração de preços baixos, a qualidade de produtos e o tempo limitado de promoção pelos sites especializados nesse tipo de comércio eletrônico.

Por se tratar algo novo, ainda há poucos conceitos na literatura científica no que diz respeito às compras coletivas on-line. Para Dornelas (2001, p 74), "é um modelo de negócios que tem como principal objetivo unir compradores, pessoas físicas ou jurídicas, e então organizar um pedido com alto volume, permitindo que empresas ou pessoas passem a ter poder de negociação de grandes compradores".

Para este estudo, adotou-se definição análoga às apresentadas, entendendo-se compra coletiva on-line como uma modalidade de comércio eletrônico que objetiva vender produtos e serviços em oferta para um número mínimo preestabelecido de consumidores. A compra coletiva é nominada dessa forma porque as ofertas somente são finalizadas se o anunciante consegue concretizar a venda de uma quantidade mínima do que foi ofertado, ou seja, a venda só ocorre se várias pessoas adquirirem as ofertas disponibilizadas. 


\subsection{Modelo Teórico}

O modelo teórico que sintetiza o objetivo deste estudo é apresentado na figura 1. De forma resumida, o modelo estabelece a relação entre os construtos compra por impulso, interesse em compras coletivas on-line e insegurança em relação ao site e a variável intensidade de compras coletivas on-line. Cada construto e variável será definida a seguir, bem como será discutida e formalizada a relação entre as variáveis, resultando em suas referidas hipóteses. Antecipando, entretanto, tais relações, o modelo teórico ilustra a relação entre essas variáveis de forma a facilitar o entendimento do leitor sobre o propósito deste trabalho. Esses construtos foram escolhidos por apresentarem uma relação primária com a variável dependente. Dito de outra forma, dada a natureza exploratória do trabalho, o modelo prioriza a construção de conhecimento baseado em construtos que, teoricamente, têm uma forte relação com a variável dependente analisada.

Figura 1 - Modelo de análise proposto

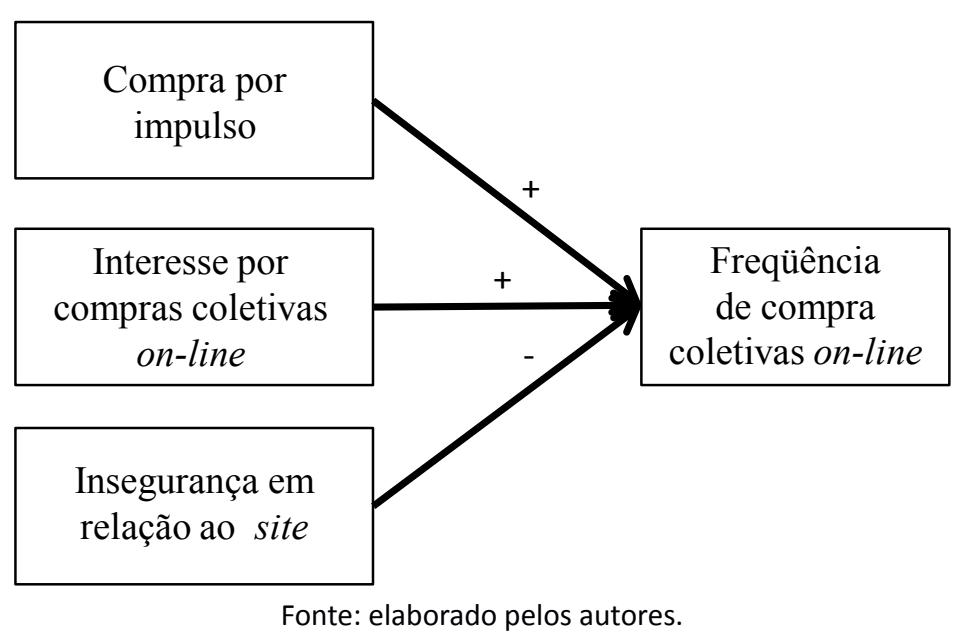

\subsubsection{Compra por Impulso}

Uma compra por impulso é entendida como um comportamento não planejado, muitas vezes oriundo de um desejo incontrolável e persistente de adquirir algum bem de forma instantânea. Nesse sentido, a real necessidade não é conhecida antes da entrada no ambiente de loja, porque essa compra é diferente da compra tradicional, cuja necessidade da compra já é previamente conhecida pelo indivíduo e apenas lembrada no ambiente da loja (BEATTY e FERREL, 1998).

Alguns consumidores realizam a compra impulsiva na busca apenas de um prazer, visto que o bem comprado passa ser um bem secundário. Percebe-se, assim, uma correlação entre as incidências de compra por impulso e satisfação das necessidades de estima e autorrealização a partir da compra, que acaba sendo geradora de contentamento e satisfação, conforme o pensamento de Hausman (2000). Costa e Larán (2003; 2006), após vasta revisão de literatura, propuseram um modelo integrativo do comportamento de compra impulsiva no campo do comércio eletrônico, contemplando as variáveis de impulsividade, ambiente, compra por impulso, circulação, emoções positivas e emoções negativas, bem como as relações entre elas, constando que a impulsividade individual do consumidor foi o que se destacou como antecedente de maior impacto na compra por impulso. Como concluíram os autores, "a impulsividade do consumidor não é expressa apenas sob a forma de atos de compra com reduzida capacidade avaliativa, mas 
também como uma incapacidade momentânea de postergar recompensas com intuito de satisfação imediata" (COSTA E LARÁN, 2003, p. 44).

Baseado nessas discussões, este estudo define compra por impulso como um comportamento que leva uma determinada pessoa a comprar produtos e serviços sem uma necessidade imediata de uso ou casos em que o produto ou serviço comprado não será utilizado.

\subsubsection{Interesse por Compras Coletivas On-line}

O conceito de interesse por compras coletivas on-line ainda está em processo de desenvolvimento, de modo que não foi encontrada na literatura nenhuma escala para medir o interesse por compras coletivas on-line. Pode-se afirmar, entretanto, que esse conceito está diretamente relacionado com as redes sociais virtuais, que é uma estrutura formada por atores (amigos) e suas relações interpessoais (ORTIZ e outros, 2004).

Em trabalho realizado por Marteleto (2001), que analisou o fluxo de informações dentro de uma rede social do subúrbio do Rio de Janeiro, redes sociais foram definidas como "um conjunto de participantes autônomos, unindo ideias e recursos em torno de valores e interesse compartilhados". Atores ou participantes, neste contexto, são caracterizados como "pessoas que se comunicam em uma dada rede" (MARTELETO, 2001, p.75). Para este estudo, o conceito de redes sociais é entendido como um conjunto de atores interligados e interagindo entre si, com objetivos em comum.

Nesse contexto, ressalta-se que as redes sociais são dinâmicas, fazendo com que os atores possam criar novas relações de acordo com as suas necessidades. Conforme com Lopes e Baldi (2009, p. 1009), "uma rede não é algo dado, mas em constante construção, o que permite aos atores construírem redes alternativas que possibilitem maior autonomia". É nesse dinamismo de relações que uma grande variedade de informações é trocada entre os amigos, constituindo uma poderosa ferramenta de comunicação. "É sabido que as redes sociais desempenham um papel importante na disseminação de ideias e informações" (NARAYANAM e NARAHARI, 2011, p. 56).

Como dito anteriormente, poucos estudos desenvolveram uma definição conceitual para o construto interesse por compras coletivas on-line. Uma das razões para a falta de uma definição talvez seja o fato de que o fenômeno compras coletivas on-line tenha surgido somente nos últimos dois anos. Neste estudo, o interesse por compras coletivas on-line é definido como a vontade ou o desejo de uma pessoa de procurar e realizar compras coletivas ofertadas por empresas no âmbito da internet.

\subsubsection{Insegurança em relação ao site}

Este estudo define insegurança em relação ao site como sendo a percepção de que determinado site de venda de produtos e serviços é suscetível à exposição das informações pessoais e financeiras do comprador para outros usuários da internet. Além de essa definição ser ampla, a discussão sobre a percepção do consumidor pode ter várias facetas, mas é de grande importância nos estudos relacionados ao comportamento do consumidor (OLIVEIRA, TAVARES e SATO, 2010). A definição de insegurança utilizada nesta pesquisa está relacionada com a segurança que o consumidor sente em relação ao processo de compra, que, segundo Cernev e Leite (2005), é vago e abrangente. Pode-se argumentar que segurança na internet é um conceito cujos limites são ainda tênues e que pode ser analisado por diferentes pontos de vistas. Para os internautas, a segurança do site poderia ser entendida como um adjetivo, relacionada à percepção de risco e à confiança dos usuários em relação aos ambientes, às tecnologias e aos agentes de comércio eletrônico (CE). 
A análise de Oliveira e colaboradores (2010) sugere, também, o reconhecimento da segurança como um aspecto técnico ou de infraestrutura, mas de responsabilidade dos agentes de CE.

Sob outro ponto de vista, Albertin e Moura (1998) observam que a ameaça de segurança, ou o risco, é definida como circunstância, condição ou evento com potencial de causar danos em dados ou recursos de rede, na forma de destruição, exposição, modificação de dados, negação de serviço, fraude, perda ou abuso. As ameaças de segurança de mensagem podem ser divididas em três categorias: confidencialidade (acesso restrito às informações), integridade (conteúdo permaneçe inalterado) e autenticação (identificação dos envolvidos).

Essa definição de segurança do site está, até certo ponto, relacionada com a confiança do comprador. Para Albertin e Moura (1998), confiança, no ambiente web, pode ser definida tanto como comportamento ou atitude quanto como percepção e compreende vários aspectos, incluindo segurança, privacidade, honestidade, reputação, marca, leis de proteção ao consumidor, etc. Analisando a literatura existente, Yaghoubi e colaboradores (2011) elaboraram uma comparação analítica entre 10 diferentes modelos de confiança eletrônica e concluíram que o modelo desenvolvido por McKnight e colaboradores (2002) contém todas as dimensões abordadas pelos demais pesquisadores. Este estudo considera que dois fatores estão associados à confiança: qualidade do site e reputação do vendedor.

\subsubsection{Intensidade de Compras Coletivas On-line}

Embora o comércio realizado por meio da internet não seja um fenômeno recente, as pesquisas que envolvem esse tema focam especialmente o comportamento do consumidor quanto aos fatores que influenciam sua tomada de decisão, propondo modelos para análise desse comportamento e também tipologias que classifiquem esse tipo de consumidor, tal como fazem Lokken e colaboradores, 2003; Cheung, Chan e Limayen, 2005; Liebermann e Stashevsky, 2009; Dennis e colaboradores, 2009 e, no Brasil, Miranda e Arruda, 2004.

A variável intensidade de compras tem sido pouco explorada nos estudos relacionados ao tema. Liu \& Forsythe $(2011,101)$ definem intensidade de compra como "[...] o número de categorias de produtos e a frequência de compra online durante um determinado período de tempo depois da adoção inicial do canal [de compra] online". Entende-se que, apesar de válida a definição de Liu \& Forsythe (2011), para a finalidade do presente estudo, tal conceito acaba por envolver duas dimensões diversas: o que se compra (categorias de produtos) e a frequência (número de compras realizadas por mês).

Para o objetivo desta pesquisa, optou-se por definir a variável intensidade de compra como a quantidade de vezes que determinado comprador realizou compras coletivas on-line. Essa definição tem por base a quantidade de compras coletivas on-line realizadas em um período de tempo e não a frequência com que o consumidor realiza tais compras, uma vez que a frequência de compras remete a certa regularidade no comportamento do consumidor e que o objetivo desta pesquisa não é entender a regularidade das compras, mas sim o volume de compras feitas. Essa definição está alinhada com o que Kau e colaboradores (2003), ao proporem uma categorização dos compradores on-line, estabelecem como "experiência de compra", que é o número de vezes que uma pessoa comprou um produto on-line. 


\subsection{Relação entre Compra por Impulso e Intensidade de Compras Coletivas On-line}

Liu \& Forsythe (2011), ao estudarem os direcionadores da intensidade de compra, concluíram que a satisfação de comprar influencia mais a intensidade (neste caso, entendida como frequência de compra e número de categorias de produtos) que os atributos de utilidade dos websites. Mesmo se tratando de uma definição diferente quanto à intensidade, os autores lançam questionamentos sobre duas dimensões que podem estar relacionadas à intensidade de compras: satisfação em comprar e usabilidade de websites.

No caso específico do estudo em questão, reduziu-se o construto impulso à perspectiva da satisfação gerada pelo ato de comprar. Dessa forma, tomando-se por pressuposto o fato de que a compra por impulso é um comportamento imbuído em emoção e que também gera satisfação e contentamento, conforme Hausman (2000) identificou, depreende-se que o comportamento impulsivo pode aumentar a quantidade de vezes que o consumidor compra em sites de compra coletiva.

Este trabalho segue os estudos sobre comportamento do consumidor ao argumentar que as promoções realizadas por compras coletivas on-line apresentam um apelo relativamente forte à emoção dos compradores. Nesse caso, compradores que são mais suscetíveis a decisões baseadas em emoções tendem a realizar compras coletivas on-line mais intensamente, pois têm maiores dificuldades para controlar seus sentimentos e proporcionar um maior tempo a si mesmos, de forma a reunir mais informações e fazer um julgamento mais racional sobre o produto ou serviço que estão comprando. Nesse sentido, a primeira hipótese deste estudo é a de que a compra por impulso está positivamente relacionada com a intensidade de compras coletivas on-line.

\subsection{Relação entre Interesse por Compras Coletivas On-line e Intensidade de Compras Coletivas On-line}

Dentre os objetivos em comum dos participantes das redes sociais virtuais, pode-se citar a busca por oportunidades de negócio, ou seja, o interesse desses participantes em aproveitar os benefícios que essas redes proporcionam. De acordo com Ciribeli e Paiva (2011), que estudaram a influência das redes e mídias sociais nos internautas brasileiros, é fato que os consumidores estão inseridos nas redes sociais, intercambiando informações, buscando descontos e promoções e influenciando mutuamente uns aos outros. As informações disponibilizadas são de grande confiabilidade, pois o ato de compartilhar opiniões sobre as características dos produtos e serviços é voluntário, sem interesse de uma ou outra empresa.

Há razões, portanto, para argumentar que as redes sociais virtuais possam exercer um papel de destaque no interesse e na intensidade de compras coletivas on-line devido ao seu dinamismo na troca de informações, fazendo com que as ofertas de produtos e serviços sejam visualizadas por um grande número de pessoas. Como na compra coletiva o negócio só é concretizado no momento em que um determinado número de compradores demonstra interesse, quanto mais internautas visualizarem as ofertas, maior será a possibilidade de sucesso da venda. Esse cenário explica o aumento no número de pesquisas em redes sociais antes da realização de uma compra. Em tal contexto, surge a segunda hipótese deste estudo: de que o interesse por compras coletivas on-line está positivamente relacionado com a intensidade de compras coletivas on-line.

\subsection{Relação entre Insegurança em relação ao site e Intensidade de Compras Coletivas On-line}

Conforme apresentado por Albertin e Moura (1998), uma fonte potencial de problemas é a preocupação dos usuários de comércio eletrônico com privacidade e segurança, o que poderia 
levar a uma não utilização dessa modalidade de comércio. A preocupação dos internautas com a fonte potencial de riscos gerada pelas transações eletrônicas tende a estar negativamente associada às compras coletivas on-line. A existência de elementos como, por exemplo, "vírus", que podem acessar de forma indevida informações sigilosas do comprador, também pode levar os internautas a evitarem compras coletivas on-line por receio de que suas informações pessoais sejam utilizadas de forma indevida por outras pessoas. Desse modo, para os fins deste estudo, o construto segurança pode ser entendido pelo usuário como uma percepção acerca de confiança e risco.

Considerando os fatores associados à confiança pesquisados por McKnight e colaboradores (2002) - qualidade do site nas dimensões de interface do usuário, capacidade de resposta e necessidade de preenchimento -, quanto maior for a percepção de qualidade dos compradores com relação a esses fatores, maior será a tendência de aumento do volume de compras, pois os compradores tendem a se sentir mais seguros. Por exemplo, um site de venda de produtos que não apresente o tradicional ícone com formato de "cadeado" tende a proporcionar maior insegurança ao comprador, já que tal ícone tem um significado comumente aceito pelos compradores de que o site está protegido contra a invasão de outros internautas. Diante disso, surge a terceira hipótese desta pesquisa: a de que a insegurança em relação ao site está negativamente relacionada com a intensidade de compras coletivas on-line.

\section{METODOLOGIA}

Para alcançar o objetivo deste estudo, foi utilizada uma visão positivista, baseada na técnica de levantamento survey explanatória para a coleta de dados e em procedimentos estatísticos de análise fatorial exploratória e análise de regressão logística.

\subsection{Amostra}

A população da amostra foi constituída de pessoas que tivessem acesso à internet e feito uma compra coletiva on-line no último ano anterior à participação na pesquisa. O processo de seleção dos respondentes utilizado foi o de amostragem pela técnica bola de neve, no qual cada participante indica outras pessoas para participar da pesquisa (MALHOTRA, 2001). A seleção dos respondentes ocorreu inicialmente por meio da rede de contatos dos pesquisadores envolvidos nesta pesquisa. Para participar, a pessoa precisava acessar o link de acesso ao questionário, disponibilizado por meio de um e-mail. Além disso, os participantes da pesquisa foram estimulados a enviar o e-mail recebido para os seus próprios contatos, aumentando o potencial de participantes e da amostra. O questionário ficou disponível para os respondentes durante um período de quatro dias, de 22 a 25 de julho de 2011. Ao final do período de pesquisa, 156 pessoas responderam o questionário.

\subsection{Instrumento de Coleta de Dados}

O instrumento de coleta de dados empregado foi um questionário contendo questões fechadas com múltiplas escolhas e com escalas de concordância do tipo Likert de 5 pontos. 0 valor mais baixo da escala (1) corresponde ao menor valor de concordância dos respondentes a uma determinada pergunta, enquanto que o valor mais alto da escala (5) corresponde ao maior valor de concordância. As questões foram adaptadas a partir de artigos de comportamento do consumidor, especialmente do artigo de Liebermann e Stashevsky (2009) e Koufaris (2002), que 
utilizaram a metodologia survey. A validação do instrumento de pesquisa foi realizada por dois professores e pesquisadores das área de tecnologia da informação e serviços e experientes na condução de pesquisas quantitativas feitas por meio de questionários.

\subsection{Construtos e Escalas Utilizadas}

O construto compra por impulso $(\mathrm{Cl})$ buscou avaliar o grau de impulsividade dos respondentes em relação ao seu comportamento quanto a compras on-line. Para isso, as seguintes questões foram utilizadas: CPI1 - sou propenso a fazer uma compra por impulso em sites de compras coletivas; CPI2 - compro produtos ou serviços promocionais em sites de compras coletivas mesmo que não esteja precisando; e CPI3 - já comprei algo por site de compras coletivas que acabei não utilizando.

Já o construto interesse por compras coletivas on-line (ICC) procurou avaliar o interesse pessoal do comprador em fazer compras coletivas on-line. Para isso, as seguintes questões foram utilizadas: ICC1 - cadastro-me nos sites de compras coletivas on-line indicados pelos amigos; ICC2 - alguém que eu presenteei com um cupom de compra coletiva on-line passou a realizar compras coletivas eletrônicas; e ICC3 - eu realizei uma compra coletiva on-line porque fui estimulado pelos meus amigos.

O construto insegurança em relação do site (INS), por sua vez, teve por objetivo avaliar a importância da insegurança em relação ao site para o respondente. Para tanto, foram utilizadas as seguintes questões: INS1- deixei de finalizar uma compra coletiva eletrônica porque não sinto segurança em informar meus dados em sites de internet; INS2 - estou ciente de que sites de compras coletivas podem conter vírus com potencial para acessar meus dados de forma indevida; INS3 - deixo de comprar se desconfio da segurança do site; e INS4 - deixo de comprar em sites de compra coletiva porque tenho cuidado quando vou fornecer dados pessoais.

A variável intensidade de compra coletiva on-line (INT) mede a intensidade de compra coletiva on-line de um determinado respondente. Ela é uma variável binária que mede quantas vezes, no último ano, o respondente fez esse tipo de compra. Respondentes que fizeram menos de cinco compras coletivas on-line são designados como compradores de baixa intensidade, enquanto que respondentes que fizeram mais de cinco compras coletivas on-line são considerados como compradores de alta intensidade. Por ser uma variável binária, respondentes com uma baixa intensidade de compra receberam o valor igual a zero $(0)$ e respondentes com alta intensidade de compra receberam o valor igual a um (1).

\subsection{Análises Estatísticas}

Inicialmente, foram realizadas análises preliminares com o objetivo de verificar a existência de (i) dados faltantes e (ii) outliers. Para os dados faltantes, procedeu-se a uma análise visual dos dados. Para os outliers, foram feitas análises univariadas, com o uso das distribuições de cada construto, e uma análise multivariada, com o uso da distância de Mahalanobis (TABACHNICK e FIDELL, 2007). Depois das análises preliminares, foram feitas as análises de resíduos com o objetivo de identificar casos extremos a serem eliminados.

Posteriormente, os dados foram analisados para obtenção das características da amostra, utilizando-se basicamente de informações sobre a distribuição amostral. Em seguida, foi feita uma análise fatorial exploratória, para confirmar a formação dos construtos esperados, e uma análise de confiabilidade dos dados por meio da análise de confiabilidade composta (FORNELL e LARCKER, 1981). Por fim, foi feita uma análise de regressão logística para avaliar a relação entre os construtos e a variável binária intensidade de compra coletiva on-line. Esta técnica estatística foi escolhida 
tendo como base a necessidade de avaliar a ocorrência ou não de um evento por meio de uma variável dicotômica (GUIMARÃES e NETO, 2002), que, no caso deste estudo, refere-se à intensidade de compra coletiva on-line (INT). Além disso, de acordo com o estudo realizado por Pinto e Lara (2008), esta técnica é pouco utilizada em estudos que analisam o comportamento do consumidor.

\section{RESULTADOS}

\subsection{Análises Preliminares}

Resultados das análises de dados faltantes mostraram a existência de sete respondentes com uma quantidade relativamente substancial de respostas em branco. Optou-se pela exclusão desses respondentes da amostra dado a grande quantidade de perguntas não respondidas. Os resultados das análises univariada e multivariada mostraram a existência de quatro respondentes caracterizados como outliers. Novamente, optou-se pela exclusão desses respondentes, pois os resultados relativos à distância de Mahalanobis eram significativamente inferiores ao valor de corte de 0,001 em uma distribuição de $c^{2}$ (TABACHNICK e FIDELL, 2007). Para análise de resíduos, utilizou-se aqueles resultantes do modelo completo (COHEN e outros, 2003): índice de desvio (deviance) e índice de influência (leverage). Com base nos valores de corte estabelecidos pela literatura (TABACHNICK e FIDELL, 2007; COHEN e outros, 2003; HAIR e outros, 2006), dois respondentes foram identificados como ofensores dos dados da amostra e foram excluídos. Com a exclusão desses casos considerados suspeitos, a amostra final totalizou 143 respondentes.

\subsection{Caracterização da Amostra}

Após a exclusão de 13 casos, a amostra ficou composta de 143 respondentes, todos residentes no estado do Rio Grande do Sul. De forma geral, a amostra está caracterizada da seguinte maneira: $60 \%$ dos respondentes são do sexo feminino com idade média de 32 anos, enquanto que os respondentes do sexo masculino tem idade média de 29 anos; $63 \%$ dos respondentes possuem um título de graduação ou pós-graduação; e $53 \%$ dos respondentes são solteiros.

\subsection{Resultados da Análise Fatorial Exploratória}

A análise fatorial exploratória teve o objetivo de verificar os escores fatoriais de cada variável e avaliar a formação dos construtos teorizados neste estudo. O resultado do teste de esferacidade de Barlett $\left(c^{2}=301,317\right.$, Sig. $<0,000$ ) evidenciou a adequação da amostra para a realização da técnica. Em seguida, utilizou-se a análise de componentes principais com o processo varimax de rotação para obtenção dos fatores e das cargas fatoriais de cada variável. Os resultados dessa análise são apresentados na tabela 1, em que as cargas fatoriais inferiores a 0,400 foram omitidas da tabela para facilitar a visualização e as variáveis foram agrupadas em função dos seus construtos.

A regra para a determinação do número de fatores extraídos é baseada nos autovalores iniciais cujo valor esteja acima do valor de corte usualmente adotado de 1,000 (HAIR e outros, 2006; TABACHNICK e FIDELL, 2007). Os autovalores iniciais apresentados na tabela 1 evidenciam a formação dos três construtos teorizados no estudo. Além disso, todas variáveis tiveram suas cargas fatoriais relacionadas a apenas um construto, o que significa que nenhuma variável apresentou evidência estatística de relação significativa com outros construtos. Os três fatores explicam mais de $56 \%$ da variância total das variáveis independentes utilizadas neste estudo. 
Tabela 1 - Resultados da Análise Fatorial Exploratória

\begin{tabular}{cccc}
\hline & Fator 1 & Fator 2 & Fator 3 \\
\cline { 2 - 4 } Variável & $\begin{array}{c}\text { INS } \\
\text { (Insegurança em } \\
\text { relação ao site) }\end{array}$ & $\begin{array}{c}\text { CIP } \\
\text { (Compra por impulso) }\end{array}$ & $\begin{array}{c}\text { (Interesse em compras coleti- } \\
\text { vas on-line) }\end{array}$ \\
\hline INS1 & 0,638 & & \\
INS2 & 0,650 & & \\
INS3 & 0,690 & 0,783 & \\
INS4 & 0,745 & 0,754 & 0,735 \\
CIP1 & & 0,628 & 0,753 \\
CIP2 & & & 0,655 \\
CIP3 & & & 1,096 \\
ICC1 & & 1,649 & 56,45 \\
ICC2 & & 45,49 & 0,758 \\
ICC3 & 2,900 & & 0,767 \\
Autovalor & 28,99 & & \\
\hline Confiabilidade composta & 0,776 & & \\
\hline
\end{tabular}

Fonte: elaborado pelos autores.

A tabela 1 apresenta, ainda, os resultados da confiabilidade composta, utilizada para testar a confiabilidade dos construtos. Os resultados mostram que todos os construtos apresentam resultados acima do valor de corte de 0,700 (HAIR e outros, 2006). Com base nas variáveis de cada fator, foram construídos os construtos utilizados na análise de regressão logística. Para a obtenção dos escores de cada construto, foi feita a média aritmética dos escores de cada variável que compõe um determinado construto.

\subsection{Resultados da Análise de Regressão Logística}

A tabela 2 apresenta os resultados da estatística descritiva de cada construto e da variável intensidade de compra coletiva on-line, bem como a correlação entre os mesmos. Como pode ser constatado, não existem indícios de alta colinearidade entre os construtos.

Para a análise de regressão logística, inicialmente foi feito um modelo nulo, ou seja, um modelo contendo apenas a variável dependente intensidade de compra coletiva on-line e sem nenhum construto servindo como variável independente. $O$ objetivo desse modelo é estabelecer um valor de -2log likelihood que possa ser utilizado de parâmetro para comparação em relação ao modelo completo, em que todos os construtos são inseridos como variáveis independentes, e, assim, determinar a contribuição dos construtos para melhorar o poder de explicação do modelo.

Tabela 2 - Resultados da Análise Fatorial Exploratória

\begin{tabular}{cccc}
\hline & Fator 1 & Fator 2 & Fator 3 \\
\cline { 2 - 4 } Variável & $\begin{array}{c}\text { INS } \\
\text { (Insegurança em } \\
\text { relação ao site) }\end{array}$ & $\begin{array}{c}\text { CIP } \\
\text { (Compra por } \\
\text { impulso) }\end{array}$ & $\begin{array}{c}\text { ICC } \\
\text { (Interesse em } \\
\text { compras coletivas } \\
\text { on-line) }\end{array}$ \\
\hline INS1 & 0,638 & \\
INS2 & 0,650 & \\
INS3 & 0,690 &
\end{tabular}




\begin{tabular}{cccc}
\hline Variável & Fator 1 & Fator 2 & Fator 3 \\
\cline { 2 - 4 } & $\begin{array}{c}\text { INS } \\
\text { (Insegurança em } \\
\text { relação ao site) }\end{array}$ & $\begin{array}{c}\text { CIP } \\
\text { (Compra por } \\
\text { impulso) }\end{array}$ & $\begin{array}{c}\text { ICC } \\
\text { (Interesse em } \\
\text { compras coletivas } \\
\text { on-line) }\end{array}$ \\
\hline INS4 & 0,745 & & \\
CIP1 & & 0,783 & \\
CIP2 & & 0,754 & \\
CIP3 & 0,628 & \\
ICC1 & & & 0,735 \\
ICC2 & & & 0,753 \\
ICC3 & 2,900 & 1,649 & 0,655 \\
Autovalor & 28,99 & 45,49 & 56,096 \\
\% Variância acumulada & & & 0,75 \\
Confiabilidade composta & 0,776 & 0,767 & 0,758 \\
\hline
\end{tabular}
Fonte: elaborado pelos autores.

O valor de -2log likelihood do modelo nulo é de 178,12, servindo de base, portanto, para comparação do valor encontrado no modelo completo. O valor de -2log likelihood do modelo completo é de 153,21. O resultado do teste de razão do likelihood, $c^{2}=24,30$ e df=3 (sig < 0,000), mostra que a diferença de ajuste entre o modelo nulo e o modelo completo é significativa. Essa diferença representa um pseudo- $R^{2}$ de 0,133 , o que indica que o modelo explica aproximadamente $13 \%$ da variação na intensidade de compra coletiva on-line feita pelos respondentes da amostra analisada. Por fim, o resultado do teste de Hosmer e Lemeshow $\left(c^{2}=24,30\right.$ e df=3, sig $=$ $0,190)$ mostra que o modelo proposto se ajusta bem aos dados observados. Esses resultados indicam que o modelo completo inclui construtos que contribuem significativamente para melhorar o ajuste do modelo aos dados observados, evidenciando, também, que algum(uns) construto(s) tem uma relação significativa para explicar a variação na intensidade de compra coletiva on-line.

A tabela 3 mostra os resultados dos coeficientes de regressão e os valores da razão de chances (odds ratio). De acordo com os resultados, dois construtos estão estatisticamente relacionados com a variável intensidade de compra coletiva on-line. Um dos construtos é o interesse por compras coletivas on-line, que teve um valor de significância para o teste de Wald (12.765 e sig $<0,000$ ) abaixo do valor de corte de 0,05 , confirmando a hipótese 2 (relação entre interesse por compras coletivas on-line e intensidade de compras coletivas on-line). O outro construto é a insegurança em relação ao site, que também teve a significância de seu valor estatístico (Wald $=5,256$ e sig $=0,022$ ) abaixo do valor estabelecido de corte, confirmando a hipótese 3 (relação entre insegurança em relação ao site e intensidade de compras coletivas on-line).

Tabela 3 - Resultados da Análise de Regressão Logística

\begin{tabular}{ccccccc}
\hline Variáveis & Beta & Erro-padrão & Wald & Df & Sig. & $\begin{array}{c}\text { Razão de chan- } \\
\text { ces }\end{array}$ \\
\hline Constante & $-2,175$ & 1,527 & 2,029 & 1 & 0,154 & 0,114 \\
INS & $-0,711$ & 0,310 & 5,256 & 1 & 0,022 & 0,491 \\
CIP & 0,029 & 0,256 & 0,013 & 1 & 0,910 & 1,029 \\
ICC & 1,218 & 0,341 & 12,765 & 1 & 0,000 & 3,379 \\
\hline
\end{tabular}


A análise dos resultados mostra que pessoas com maior interesse em compras coletivas (ICC) têm maiores chances de realizar compras coletivas em maior intensidade durante um ano. A razão de chances demonstra que uma unidade a mais na escala de interesse em compras coletivas on-line eleva em 3,379 a chance de uma pessoa realizar mais de cinco compras coletivas on-line em um ano.

Os resultados evidenciam, ainda, que, quanto maior a insegurança em relação ao site, menores as chances de a pessoa realizar compras coletivas em maior intensidade. De acordo com os resultados, uma unidade a mais na escala de insegurança aumenta em apenas 0,491 a chance da pessoa realizar compras coletivas em maior intensidade no ano. É importante destacar que a relação entre a insegurança quanto ao site e a intensidade de compra coletiva on-line é exponencial, de modo que à medida que a insegurança aumenta a intensidade de compra coletiva on-line diminui exponencialmente. Se a escala de insegurança em relação ao site for aumentada em duas unidades, por exemplo, a chance de aumentar a intensidade de compra coletiva on-line é de apenas $0,241(0,491 \times 0,491)$.

\section{DISCUSSÃO DOS RESULTADOS}

Os resultados encontrados proporcionam algumas informações que levam à reflexão sobre o comportamento dos compradores de compras coletivas on-line. Primeiramente, como já era esperado, ressalta-se que os consumidores com maior interesse em realizar compras coletivas on -line são aqueles que efetivamente as fazem em maior intensidade, ou seja, são os que fazem mais do que cinco compras em um ano. Esse resultado mostra que o construto interesse por compras coletivas on-line reflete, mesmo que parcialmente, um conjunto de desejos dos consumidores em utilizar o serviço de compras coletivas on-line que, de alguma forma, é realizado por meio das compras efetuadas. Ou seja, os resultados sugerem que existe uma correspondência entre o interesse de realizar compras coletivas e sua efetiva realização. Apesar de essa relação já ser esperada, a sua medição empírica permite a obtenção de um coeficiente que ajuda a determinar sua intensidade, a qual se mostra relativamente forte. Embora não possa ser utilizado como o coeficiente populacional, o coeficiente obtido já proporciona uma base para análise em futuros estudos.

O construto interesse por compras coletivas on-line introduzido neste estudo pode ajudar a determinar o desejo de um determinado comprador em serviços de compras coletivas on-line. Empresas prestadoras desse tipo de serviço podem utilizar essa informação para identificação e seleção de consumidores que estejam mais propensos a fazer compras coletivas on-line. Devido ao seu caráter exploratório, entretanto, esse construto pode ser melhorado.

Percebeu-se, também, que a insegurança que o consumidor sente em relação a um determinado site de compras coletivas on-line afeta negativamente a probabilidade desse consumidor de realizar uma grande quantidade de compras. Esse resultado vai ao encontro de outros achados semelhantes na literatura, evidenciando que os consumidores se sentem desestimulados a comprar quando não percebem que o site que faz a venda se preocupa com a proteção do comprador. Os consumidores preferem não comprar produtos e/ou serviços em oferta, preservando suas informações pessoais, a realizar uma compra que pode apresentar algum tipo de risco. Isso mostra que o valor percebido da oferta tende a ser menor que um determinado valor percebido da segurança.

Por fim, as análises não encontraram nenhum suporte para a hipótese 1 (relação entre compra por impulso e intensidade de compras coletivas on-line), que estabelece uma relação positiva entre compra por impulso e a intensidade de compra coletiva on-line. Possivelmente, os respondentes da amostra não sejam suscetíveis a incentivos que os levem a realizar esse tipo de compra. Outra razão para tal resultado pode ser o tamanho da amostra, o que limita o poder das análises estatísticas. 


\section{CONCLUSÃO}

Este trabalho mostrou a relação entre os construtos interesse por compras coletivas on -line, compra por impulso e insegurança em relação ao site e a intensidade com que o comprador fez compra coletiva on-line no último ano. Em resumo, consumidores interessados em aproveitar as compras coletivas on-line são mais propensos a realizá-las. Por outro lado, consumidores que se sentem inseguros em um site de compra coletiva on-line diminuem significativamente a quantidade de compras que fazem. Por fim, não foi encontrada nenhuma relação entre compra por impulso e intensidade de compras coletivas on-line. Esses resultados podem ser relevantes tanto para futuros trabalhos acadêmicos como para empresas interessadas em desenvolver estratégias mercadológicas que tragam ganhos mais efetivos.

Do ponto de vista acadêmico, os resultados encontrados são pioneiros no Brasil tendo em vista que este é um dos primeiros estudos feitos sobre o fenômeno de compras coletivas on-line, abrindo oportunidades para que outros trabalhos sejam desenvolvidos e o conhecimento sobre o tema seja aprofundado. Uma das limitações deste estudo é a quantidade reduzida de construtos analisados no modelo. Futuras pesquisas podem investigar a influência de novos construtos na intensidade de compra coletiva on-line, como, por exemplo, o tamanho das redes sociais virtuais de cada consumidor.

Outra contribuição deste estudo é a utilização de construtos e a investigação empírica do modelo proposto. Os construtos utilizados foram escolhidos baseados na sua relação com a variável dependente, de forma que suas definições e seus instrumentos de medição constituem, de certo modo, uma contribuição importante para a literatura sobre compras coletivas on-line. A contribuição proporcionada por este estudo foi a de entender, no contexto da pesquisa, como pensa e age o consumidor. Durante esse processo de construção, entretanto, algumas questões são levantadas para futuros trabalhos. A primeira diz respeito ao construto interesse por compras coletivas on-line, uma vez que as escalas utilizadas para capturá-lo precisam ser melhoradas. Outra limitação diz respeito ao tamanho da amostra e ao processo de seleção dos respondentes, o que impede a generalização dos resultados para outras populações. Por fim, futuros estudos podem explorar outras variáveis dependentes que contribuam para um entendimento mais amplo sobre o comportamento do consumidor de compras coletivas on-line.

No campo executivo, este trabalho pode servir para gerentes interessados em desenvolver estratégias e ações mercadológicas com maiores chance de sucesso para o seu público-alvo. O construto interesse em compras coletivas on-line, por exemplo, pode ser usado para identificar e selecionar consumidores com maior potencial de compra. Além disso, empresas de compras coletivas on-line devem trabalhar não apenas para proporcionar melhores ofertas, mas também para oferecer segurança aos consumidores. Os consumidores que se sentem mais seguros tendem a fazer compras coletivas on-line em maior intensidade. Assim, informações obtidas neste estudo podem ser utilizadas para que esse fenômeno continue avançando no mercado consumidor brasileiro. 


\section{REFERÊNCIAS}

ABREU, E.; SOUSA, P. V. Um mundo de descontos: proposições acerca dos sites de compras coletivas. Culturas Midiáticas, ano IV, n. 06, jan- jun. 2011

ALBERTIN, A. L.; MOURA, R.M. Comércio eletrônico: seus aspectos de segurança e privacidade. Revista de Administração de Empresas, v. 38, n.2, abr/jun 1998.

BEATTY, S.; FERREL, E. Impulse buying: modeling its precursors. Journal of Retailing, $\mathrm{v}$. 74, n. 2, 1998.

CERNEV, A. K; LEITE, J. C. Segurança na Internet: a percepção dos usuários como fator de restrição ao comércio eletrônico no Brasil. Enanpad, Brasília. Anais do Enanpad, 2005.

CHEUNG, C. M. K; CHAN, G. W. W.; LIMAYEM, M. A critical review of online consumer behavior: empirical research. Journal of Electronic Commerce in Organizations. v. 3, n. 4, p. 1-19, 2005.

CIRIBELI, João P., PAIVA, Victor H. P. Redes e Mídias Sociais na Internet: Realidades e Perspectivas de um Mundo Conectado. Revista Mediação, v. 13, n. 12, janeiro/junho, 2011.

COHEN, J.; COHEN, P.; WEST, S.G, AIKEN, L.S. Applied Mutiple Regression/Correlation Analysis for the Behavioral Sciences. 3rd Ed. Mahwah, NJ: Lawrence Erlbaum Associates, 2003.

COLDIBELI, L. Donos de sites de compras coletivas mudam estratégia para crescer. $\mathrm{Uol}$ Notícias, São Paulo, 28 ago. 2012. Disponível em: http://economia.uol.com.br/ultimasnoticias/redacao/2012/08/29/ ex-donosde-sites-de-compras-coletivas-mudamestrategia-para-crescer.jhtm. Acesso em 01 set. 2012.

COSTA, F. C. X. ; LARAN, Juliano Aita. A Compra por impulso em ambientes on-line. RAE.
Revista de Administração de Empresas, São Paulo, v. 43, n.3, 2003. Influências do ambiente de loja e o comportamento de compra por impulso: a visão dos clientes de lojas virtuais. RAUSP. Revista de Administração, São Paulo, v. 41, n.5, p. 96-106, 2006.

DENNIS C.; MERRILEES B.; JAYAWARDHENA C.; WRIGHT L T. e-Consumer Behaviour. European Journal of Marketing, v. 43, n. 9/10, p. 11211139, 2009.

DHOLAKIA, U. M., How Effective are Groupon Promotions for Businesses? (september 28, 2010). Disponível em: http://ssrn.com/ abstract=1696327. Acesso em 15 jul. 2011.

DORNELAS, J.C.A. Empreendedorismo: transformando ideias em negócios. Rio de Janeiro: Campus, 2001.

E-bit. Dados estatísticos sobre a internet e o comércio eletrônico. Disponível em: http://ecommerce.org.br/stats.php. Acesso em 27 julho. 2011.

EDELAM, B. G., JAFFE, S.; KOMINERS, S. D., To Groupon or Not to Groupon: The Profitability of Deep Discounts (June 16, 2011). Harvard Business School NOM Unit Working Paper, No. 11-063. Disponível em: http://ssrn.com/ abstract=1727508. Acesso em 15 jul. 2011.

FAUST, A. A histeria das compras coletivas. Revista Exame, São Paulo, 22 dez. 2010. Disponível em: http://exame.abril.com.br/ noticia/a-histeria-das-compras-coletivas. Acesso em 29 maio 2011.

FEIJÓ, B. V. Tudo fica a preço de banana nos sites de compras coletivas. Exame PME, São Paulo, 24 maio 2010. Disponível em: http:// exame.abril.com.br/noticia/tudo-a-preco-debanana. Acesso em 29 maio 2011. 
FELIPINI, D. A consolidação do mercado de compras coletivas. eCommerceorg, 15 jun. 2011. Disponível em: http://e-commerce.org. br/artigos/compra-coletiva-consolidacao.php. Acesso em 01 set. 2012.

FORNELL, C. e LARCKER, D. F. Evaluating structural equation models with unobservable variables and measurement error. Journal of Marketing Research, v.18, p. 39-50, 1981.

FRONZA, J. G.; FREIRE, R. Compras Coletivas: Motivações e Implicações na Perspectiva Organizacional e dos Consumidores. In: Congresso de Ciências da Comunicação na Região Sul, 13, 2012. Anais eletrônicos... Chapecó: Unochapecó, 2012. Disponível em: http://www.intercom.org.br/papers/ regionais/sul2012/resumos/R30-1264-1.pdf. Acesso em: 10 set. 2012.

GAUDÊNCIO, J. C. C. O.; TORRES, L. M. L. S.; TORRES, R. O. M.; FIDELIS, R. A.; QUEIROZ, J. V. Como uma análise econômica em sites de compras coletivas pode servir de pesquisa de mercado. Linguagem Acadêmica, v. 1, n. 2, jul.dez. 2011, p. 219-237.

GUIMARÃES, I. A.; NETO, A. C. Reconhecimento de padrões: metodologias estatísticas em crédito ao consumidor. RAE-eletrônica, v. 1, n. 2, jul.-dez. 2002, p. 1-14.

HAIR, J.F.Jr; BLACK, W.C.; BABIN, B.J.; ANDERSON, R.E.; TATHAM, R.L. Multivariate Data Analysis. 6th ed. Upper Saddle River, NJ: Prentice Hall, 2006.

HAUSMAN, A. A multi-method investigation of consumer motivations in impulse buying behavior. Journal of Consumer Marketing, v. 17, p. 403-19, 2000.

KOUFARIS M. Applying the technology acceptance model and flow theory to online consumer behavior. Information System Research 13 2, pp. 205-223, 2002.

LIEBERMANN, Y; STASHEVSKY, S. Determinants of Online Shopping: Examination of an early- stage Online Market. Canadian Journal of Administrative Sciences, v. 26, n.4, p. 316-331, 2009.

LOKKEN, S. L.; CROSS, G. W.; HALBERT, G. L.; DERBY, C.; STANFORD, C. Comparing online and non-online shoppers. International Journal of Consumer Studies. v. 27, n. 2, mar., p. 126-133, 2003.

LOPES, F. D.; BALDI, M. Redes como perspectiva de análise e como estrutura de governança: uma análise das diferentes contribuições. RAP - Rio de Janeiro, 43(5):1007-1035, set./out. 2009.

KAU, A. K.; TANG, Y. E.; GHOSE, S. Typology of online shoppers. Journal of Consumer Marketing, v. 20, iss: 2, p.139-156, 2003.

MALHOTRA, N. K. Pesquisa de Marketing: uma orientação aplicada. 3. ed. Porto Alegre: Bookman, 2001.

MARTELETO, R. M. Análise de redes sociais - aplicação nos estudos de transferência de informação. Ci. Inf., Brasília, v. 30, n. 1, p. 7181, jan./abr. 2001.

MCKNIGHT, H. CHOUDHURY, V. e KACMAR, C. The impact of initial consumer trust on intentions to transact with a web site: a trust building model. The Journal of Strategic Information Systems, vol.11, n.3-4, dec 2002.

MIRANDA, C., ARRUDA, D. M. O. E-produto e variáveis comportamentais determinantes de compra no varejo virtual: um estudo com consumidores Brasileiros. Read - ed. 37, vol. 10, jan-fev, 2004.

NARAYANAM, R.; NARAHARI, Y. Topologies of strategically formed social networks based on a generic value function - Allocation rule model. Social Networks, 33, 56-69, 2011.

OLIVERIA, Braulio. TAVARES, Gracieli E. R. M. SATO, Karla S. Percepção: uma caixa preta para marketing. Rev. Adm. UFSM, v. 3, n. 3, p. 424 430, set./dez. 2010. 
ORTIZ, Maria G. R. HOYOS, José R. C. LOPEZ, Maria G. R. The social networks of academic performance in a student context of poverty in Mexico. Social Networks, 26, 175 - 188, 2004.

PINTO, Marcelo R. LARA, José E. O que se publica sobre o comportamento do consumidor no brasil, afinal. Rev. Adm. UFSM, v. 1, n. 1, p. 85 100, jan./abr. 2008.

PORTAL HSM. Por que as compras coletivas estão em alta no Brasil? São Paulo, 04 fevereiro 2011. Disponível em: <http://www.hsm. com.br/editorias/compras/porque-comprascoletivas-estao-em-alta-no-brasil>.. Acesso em 01 maio 2011.

ROOK, D. W. The buying impulse. Journal of Consumer Research, v. 14, p. 189-99, Sept., 1987.

STEINER, C. The next web phenom. Forbes.com, United States, 03 sep. 2010. Disponível em: http://www.forbes.com/global/2010/0913/ entrepreneurs-andrew-mason-grouponbargains-web-sensation.html. Acesso em 03 maio 2010.

TABACHNICK, B.G.; FIDELL, L.S. Using Multivariate Statistics. 5th Ed. Boston, MA: Pearson, 2007.

YAGHOUBI, N. M. KHANI, R. Y. e ESMAEALI, M. J. Trust Models in e-business; AnalyticalCompare approach. Interdisciplinary Journal of Contemporary Research in Business, vol.2, n.9, jan, 2011.conteúdo qualitativa pode facilitar a busca de respostas para as questões apresentadas? 